\title{
Encuesta sobre abuso de alcohol y medicamentos en adolescentes de cuatro escuelas del conurbano bonaerense
}

\author{
Survey on alcohol and medicine abuse among adolescents at \\ four schools in Greater Buenos Aires
}

\author{
Dra. Silvia Cabrerizo ${ }^{a, c}$, Lic. Silvana Varelab y Dra. María Inés Lutzc
}

a. Centro Nacional de Intoxicaciones, Servicio de Toxicología.

b. Sección Medicina Basada en la Evidencia, Departamento Materno-infantil.

c. Unidad Pediátrica Ambiental, Servicio de Pediatría.

Hospital Nacional

Prof. Alejandro Posadas.

Correspondencia: Dra. Silvia Cabrerizo: silviacabrerizo@yahoo. com.ar

\section{Financiamiento:}

El presente trabajo de investigación fue realizado con el apoyo de una Beca "Ramón Carrillo-Arturo

Oñativia", categoría Iniciación, otorgada por el Ministerio de Salud de la Nación, a través de la Comisión Nacional Salud Investiga.

\section{Conflicto de intereses:} Ninguno que declarar.

Recibido: 29-10-2012 Aceptado: 25-6-2014

\section{RESUMEN}

Introducción. El consumo de alcohol y medicamentos no prescritos en los adolescentes es un problema creciente en los servicios de toxicología. La "jarra loca" es una mezcla de bebidas alcohólicas y medicamentos que utilizan los jóvenes como modalidad de abuso. En los últimos años, además de psicofármacos, los jóvenes mezclan hipoglucemiantes, lo cual puede generar secuelas neurológicas o producir la muerte, dependiendo de la dosis ingerida y el tiempo de duración de la hipoglucemia.

Objetivo. Estimar la prevalencia de consumo de alcohol y medicamentos en adolescentes de $12 \mathrm{a}$ 18 años de edad que concurren a cuatro escuelas del partido de Tres de Febrero, de la provincia de Buenos Aires.

Material y métodos. Estudio observacional, descriptivo y transversal. Se realizó una encuesta autoadministrada sobre consumo de alcohol y medicamentos en adolescentes de 12 a 18 años, de cuatro escuelas de la provincia de Buenos Aires. Se realizaron talleres para intercambio de conocimientos y experiencias con los alumnos y se entregó material gráfico informativo.

Resultados. Se realizaron 977 encuestas. El 49\% de los alumnos encuestados refirió consumir bebidas alcohólicas al salir con sus amigos. El $8 \%(n=78)$ de los alumnos refirieron consumir "jarra loca" y el $49 \%$ refirieron que sus amigos lo hacían. Veintiún alumnos requirieron atención médica, al menos, en una oportunidad luego de haber tomado bebidas alcohólicas y 17 de estos habían consumido "jarra loca". El 55\% de los alumnos desconocían el uso de las pastillas que mezclan en la "jarra loca". El 48\% de los padres desaprueba el consumo de alcohol.

Conclusión. El $49 \%$ refirió tomar bebidas alcohólicas. En nuestro trabajo, el 8\% de los alumnos encuestados consumieron o probaron alguna vez "jarra loca", aunque el $49 \%$ afirmaron que sus amigos sí lo hacían.

Palabras clave: abuso de sustancias, alcohol, medicamentos bajo prescripción.

http:/ /dx.doi.org/10.5546/aap.2014.504

\section{INTRODUCCIÓN}

El consumo de alcohol y medicamentos no prescritos es un flagelo cada vez mayor entre los adolescentes. Para muchos de ellos, el consumo de bebidas alcohólicas mezcladas con medicamentos supone una forma de socializarse, sin registro de la percepción de riesgo.$^{1-5}$ Esto se presenta con distintas modalidades en distintas ciudades del mundo..$^{6-9}$ Petroianu y col. señalan que hasta un $12 \%$ de estudiantes de Medicina de la Universidad Federal de Minais Gerais, Brasil, consumen sedantes; ${ }^{6}$ Romero y col. refieren que un $6,9 \%$ de estudiantes de la Pontificia Universidad Católica de Chile consumen benzodiacepinas y $5,8 \%$, antidepresivos sin prescripción. ${ }^{7}$ También Caamaño Isorna y col. revelan una alta ingesta de medicamentos entre estudiantes universitarios españoles, la mayoría sin prescripción médica, como otra forma de policonsumo de drogas. ${ }^{8}$

La mezcla de alcohol y medicamentos se conoce como "jarra loca" o "cóctel". 1,2,5,10 Entre los medicamentos más utilizados, se encuentran las benzodiacepinas ${ }^{11,12}$ y su asociación con alcohol potencia los efectos depresores de ambos tóxicos. ${ }^{11,12}$ En los últimos años, se registraron casos de consumo de fármacos hipoglucemiantes (sulfonilureas) para lograr el efecto de "obnubilación" característico de la hipoglucemia. ${ }^{10}$ Uno de los riesgos que implica mezclar comprimidos en la "jarra" lo constituye la imposibilidad de calcular la dosis ingerida por los pacientes. En algunos casos, esta mezcla resultó en intoxicaciones graves, algunas de evolución fatal y otras que dejaron secuelas neurológicas irreversibles. ${ }^{10}$

Nos propusimos estimar el perfil de consumo de alcohol y 
medicamentos en adolescentes de 12 a 18 años que concurren a escuelas del Gran Buenos Aires, tanto a nivel público como privado, y realizar una intervención de educación para la salud para promover la concientización de los riesgos que implica la mezcla de alcohol y medicamentos con fines de abuso.

\section{OBJETIVOS}

- Estimar la prevalencia del consumo de alcohol con medicamentos ("jarra loca") en adolescentes de 12 a 18 años que concurren a cuatro escuelas, públicas y privadas, del partido de Tres de Febrero, de la provincia de Buenos Aires.

- Describir las fuentes a partir de las cuales los adolescentes acceden a medicamentos utilizados como forma de abuso, las consecuencias clínicas que presentan los adolescentes luego de combinar alcohol y medicamentos, y su opinión respecto a qué piensan sus padres sobre su consumo de alcohol.

\section{POBLACIÓN Y MÉTODOS}

El estudio se llevó a cabo desde junio de 2010 hasta mayo de 2011. La población en estudio se conformó con los alumnos de entre 12 y 18 años de edad de dos escuelas públicas y dos escuelas privadas, acordadas con las autoridades de Educación del distrito de Tres de Febrero, de la provincia de Buenos Aires.

Diseño: Trabajo observacional, descriptivo, transversal.

Instrumento o técnica de recolección de datos: Se elaboró una encuesta. Se realizó una prueba piloto de 50 encuestas y se realizaron las correcciones correspondientes previas al inicio del estudio. La encuesta autoadministrada (ver Anexo) se aplicó a los alumnos enrolados en el estudio, y se buscó incidencia de consumo de alcohol y medicamentos, frecuencia y cantidad del consumo, edad de inicio en el consumo de alcohol, fuentes de accesibilidad a los medicamentos, lugar de consumo habitual, presencia de síntomas de intoxicación, necesidad de haber recibido atención médica como consecuencia del consumo de alcohol y medicamentos, y estimación del alcohol asociado a la diversión (naturalización del consumo de alcohol).

Se consideró consumo de alcohol al consumo de, al menos, una lata de cerveza $(330 \mathrm{ml})$ o 1 vaso de vino $(200 \mathrm{ml})$ o 1 trago $(30-50 \mathrm{ml})$ de fernet, ron, ginebra, vodka, whisky, ya que estas son medidas equivalentes en gramos de alcohol que pueden dar síntomas de intoxicación etílica aguda. ${ }^{8}$

Como actividad complementaria, se realizaron talleres con los alumnos de cada curso. En estos, se presentó un caso disparador que brindaba información de cómo actuar frente a una intoxicación alcohólica aguda y consumo de medicamentos. En un segundo encuentro con los alumnos, se realizó la devolución de los resultados y se entregó material gráfico informativo.

\section{Consideraciones éticas}

El estudio contó con la aprobación del Comité de Docencia y de Bioética del Hospital Nacional Prof. A. Posadas. Antes de la realización del estudio, se solicitó a los padres o tutores el consentimiento para que sus hijos participaran y el asentimiento del alumno previo a su participación.

\section{Análisis estadísticos}

La variable de resultado final consumo de alcohol y pastillas se presentó como medidas de frecuencia al igual que las variables secundarias, como frecuencia y cantidad del consumo, edad de inicio en el consumo de alcohol, fuentes de accesibilidad a los medicamentos, lugar de consumo habitual, presencia de síntomas de intoxicación, necesidad de haber recibido atención médica como consecuencia del consumo de alcohol y medicamentos. También se evaluaron características sociodemográficas, como edad, sexo y tipo de escuela, que para su mejor entendimiento se presentaron como variables cualitativas dicotomizadas a través de porcentajes. Se compararon las variables principales entre los grupos consumidores y no consumidores a través de tests paramétricos o no paramétricos, según lo adecuado para cada comparación en particular, y se consideró una $p$ estadísticamente significativa $<0,05$.

\section{RESULTADOS}

Se realizaron un total de 977 encuestas. Las características basales de la población se muestran en la Tabla 1. La mediana de edad fue de 15 años, con un rango de entre 12 y 20 años. De acuerdo con los datos de las encuestas realizadas, el $49 \%(\mathrm{~N}=480)$ de los alumnos refirió tomar bebidas alcohólicas, 11\% refirió tomar bebidas energizantes y un $40 \%$ refirió tomar bebidas sin alcohol. Entre las bebidas alcohólicas, 5\% refirió consumir vino y cerveza; $11 \%$, bebidas de alta graduación alcohólica, como vodka, ginebra, 
ron, tequila, fernet o licores; y un 33\%, mezcla de bebidas alcohólicas cuando salían habitualmente con sus amigos. Al preguntar con qué frecuencia consumían alcohol, la prevalencia de consumo de alcohol aumentó a un $61 \%$.

E1 $8 \%(n=78)$ de los alumnos refirieron consumir mezcla de alcohol y medicamentos o "jarra loca" y el 49\% (n/N=480/972) afirmaron que sus amigos sí lo hacían.

El 33\% (n=297) refirieron haber presentado síntomas luego de haber tomado alcohol. De ellos, 251 (28\% del total) refirieron presentar mareos, náuseas, vómitos, cefalea y/o dolor abdominal y el 5\% (n= 46), síntomas graves, como pérdida de conciencia y/o convulsiones.

Veintiún alumnos refirieron requerir atención médica, al menos, en una oportunidad luego de haber tomado bebidas alcohólicas y 17 de estos refirieron haber consumido "jarra loca".

En cuanto a la respuesta de los adolescentes respecto a la opinión de sus padres, el 20\% $(n=173)$ de los padres aprobaba el consumo de alcohol, el $48 \%(n=424)$ lo desaprobaba, el $7 \%$ $(n=62)$ refirieron que sus padres desconocían que bebían alcohol y, en el $25 \%$ de los casos $(n=211)$, sus padres no opinaban al respecto.

En las escuelas privadas, los adolescentes refirieron probar alcohol más frecuentemente en el rango de edad menor de 13 años, mientras que, en la escuela pública, el mayor porcentaje (81\%) se presentó en el rango mayor de 13 años, diferencia estadísticamente significativa $\left(\mathrm{Chi}^{2}\right.$ : 74,51; p: 0,000).

Al evaluar el conocimiento del uso de las pastillas utilizadas en la "jarra loca" (N: 913), el $55 \%$ de los alumnos lo desconocía, 13\% afirmó que se trataba de psicofármacos, $7 \%$ analgésicos, $19 \%$ mezcla de comprimidos y $7 \%$ mezcla que incluía hipoglucemiantes.
Respecto de las fuentes de acceso que tenían los adolescentes a los medicamentos en la "jarra loca", el 15\% (n=96) refirió haberlos comprado, un $6 \%(\mathrm{n}=39)$ que se los habían convidado, el $5 \%(n=33)$ que los habían obtenido de su casa o de la casa de amigos y el $71 \%(n=448)$ refirió haberlos conseguido de otra manera o desconocían su origen.

De los 78 alumnos que refirieron consumir alcohol y medicamentos, "jarra loca", 62 (79\%) asistían a escuelas públicas (Chi²: 16,49; p: 0,000).

Respecto a la edad de los alumnos que refirieron el consumo de "jarra loca" (N: 78), 69 eran mayores de 15 años (Chi'2: 25,85; p: 0,000).

El $54 \%$ de los alumnos que concurrían a las escuelas públicas $(168 / 310)$ refirieron consumir durante el fin de semana más de una lata de cerveza $(330 \mathrm{ml})$ o 1 vaso de vino $(200 \mathrm{ml})$ o 1 trago $(30-50 \mathrm{ml})$ de fernet, ginebra, vodka o whisky en comparación con el $43 \%(55 / 128)$ de los que concurrían a escuelas privadas; estos datos fueron estadísticamente significativos (Chi²: 4,56; p: 0,033).

Los alumnos que refirieron consumir alcohol (N: 480) expresaron que sus motivos fueron para compartir con sus amigos (35\%), como diversión (22\%), para evadir problemas (14\%), $10 \%$ desconocía por qué consumía y un 19\% respondió más de uno de estos motivos.

Al preguntar si podían salir, reunirse con amigos y divertirse sin tomar alcohol, el 92\% (n= 894) respondió afirmativamente.

Respecto a los lugares donde los alumnos consumían habitualmente, el 30\% (n=181) refirió hacerlo en su casa o en casa de amigos; el 32\% $(\mathrm{n}=191)$, en los boliches o espacios públicos; y el $38 \%(n=230)$, en más de un lugar.

TABLA 1. Características basales de la población

\begin{tabular}{ll}
\hline Edad (años) & $12-14: 36 \%(352)$ \\
n/N: $972 / 977$ & $>15: 64 \%(620)$ \\
Sexo & Femenino: 57\% (559) \\
N: 977 & \\
Escuela & Pública: $58 \%(564)$ \\
n/N: $975 / 977$ & Privada: $42 \%(411)$ \\
Consumo de alcohol & Sí: $49 \%(480)$ \\
n/N: $972 / 977$ & \\
Consumo de alcohol y medicamentos & Sí: $8 \%(78)$ \\
n/N: $976 / 977$ &
\end{tabular}

(El n varía debido a que no todos los alumnos contestaron todos los ítems). 


\section{DISCUSIÓN}

A partir de estos datos, parecería haber un inicio temprano del consumo de "jarra loca", que comienza en la etapa escolar y podría incrementarse a edades mayores, pero esta hipótesis debe ser corroborada con un estudio de tipo analítico. La importancia de realizar prevención en este aspecto radica en que los adolescentes carecen de la percepción de riesgo que implica el consumo de alcohol y medicamentos para poder prevenir consecuencias graves para su salud.

Si bien un $49 \%$ refirió tomar bebidas alcohólicas habitualmente cuando se reunía con sus amigos, la prevalencia de consumo de alcohol aumentó a un $61 \%$ al preguntar con qué frecuencia tomaban. Este incremento se podría interpretar por los jóvenes que tomaban una vez al mes y que representaban el 13\% $(n=129)$, quienes no consideraban que consumían habitualmente.

Estos resultados son comparables con datos nacionales que evidencian el aumento del consumo de bebidas alcohólicas de un 40,7\% a un $61,9 \%$ de 2005 a 2007, respectivamente, comportamiento que ocurre tanto en varones como en mujeres. ${ }^{13}$ También evidenciamos, como en estudios nacionales, el consumo abusivo o riesgoso de alcohol durante los fines de semana, ya que es cuando existe mayor tolerancia familiar y del entorno. ${ }^{13-17}$

Cabe destacar que solo el $48 \%$ de los padres desaprueba el consumo de alcohol, por lo que políticas de educación para la salud deberían estar dirigidas a involucrar más a los adultos para disminuir el consumo de alcohol en los jóvenes.

Parecería haber un cambio en el patrón de la ingesta de bebidas alcohólicas por los adolescentes: del modelo vitivinícola mediterráneo, en el que el consumo estaba relacionado con el ritual dentro de lo familiar y alimentario, a una forma anglosajona, en la que el consumo es esporádico, pero que apunta a la intoxicación aguda ${ }^{17}$ y se prefieren bebidas destiladas, como el tequila y el vodka, como se ve reflejado en nuestros resultados.

Respecto al consumo de "jarra loca", podría haber un subregistro, tal vez por temor de revelar esta información en el ámbito escolar, a diferencia del alcohol, que no es percibido como droga por los adolescentes. Esto es una constante observada en los talleres realizados con los alumnos, también reflejada en trabajos nacionales realizados en escuelas secundarias, que evidencian que los jóvenes no consideran el abuso de alcohol como un posible problema. ${ }^{16}$

La mayoría de los adolescentes desconocen la acción farmacológica de las pastillas que consumen junto con bebidas alcohólicas y uno de los mayores riesgos detectados es que un 6\% refirieron que usaban hipoglucemiantes o mezcla de pastillas que incluyen hipoglucemiantes.

Nuestro estudio pone de manifiesto el fácil acceso y la falta de conciencia de parte de los jóvenes en cuanto al uso de medicamentos al consumirlos junto con bebidas alcohólicas. En nuestra experiencia, en el Centro Nacional de Intoxicaciones, la mayoría de las consultas por consumo de "jarra loca" que requirieron atención médica involucró a jóvenes de entre 18 y 25 años, lo que coincide con estudios nacionales. Algunos casos registrados evolucionaron al óbito y otros dejaron secuelas neurológicas asociadas al uso de hipoglucemiantes..$^{10}$ Cabe destacar que la mayoría de los jóvenes que refirieron requerir atención médica por síntomas graves habían consumido "jarra loca".

Como limitación, podemos mencionar que la muestra fue seleccionada por conveniencia a partir de una reunión con autoridades del distrito escolar, que citaron a los directores, y los que asistieron a esa reunión fueron los que participaron. Se desconocen las problemáticas en otras instituciones de la zona, a las que no se pudo acceder, lo que imposibilita la generalización de los resultados. Otra limitación está dada por el sesgo que implica toda encuesta autoadministrada.

Una fortaleza de nuestro trabajo es que el $100 \%$ de los alumnos presentes aceptó participar y estuvo predispuesto a recibir los informes de la encuesta y devoluciones que se le hicieron sobre el tema. Cabe destacar que la realización de los talleres con los alumnos dio lugar a que relataran sus experiencias y reflexiones acerca de situaciones de riesgo que ellos o sus amigos habían experimentado bajo los efectos del alcohol. También el cuerpo docente agradeció el acercamiento del equipo de salud a la escuela, lo que mostró la necesidad de abordar en forma conjunta esta problemática.

Debido al tipo de diseño, no se pudieron determinar los factores que llevan a los adolescentes a tomar la decisión de mezclar bebidas alcohólicas con medicación, así como tampoco abordar ciertas condiciones familiares o personales que los llevarían a esta situación, que debería explorarse a través de un diseño 
de investigación de mayor complejidad que los objetivos que abarca este estudio.

Este trabajo pone en evidencia que esta problemática estaría instalada en los estudiantes secundarios, por lo que sería importante intervenir sistemáticamente en este grupo.

\section{CONCLUSIÓN}

E1 51\% de los alumnos encuestados refirió no tomar alcohol. El 49\% restante refirió tomar bebidas alcohólicas habitualmente cuando se reunía con sus amigos, pero la prevalencia de consumo de alcohol aumentó a un $61 \%$ al preguntar con qué frecuencia tomaban. El 8\% consumía o había probado "jarra loca" y el 49\% refirió que sus amigos también lo hacían. El $5 \%$ de los alumnos que presentaron síntomas relacionados con el consumo de alcohol presentó pérdida de conciencia y/o convulsiones.

\section{REFERENCIAS}

1. Narváez A. Los jóvenes y la noche. Buenos Aires: Sociedad Argentina de Pediatría; 2011. Disponible en: http://www. sap.org.ar/docs/institucional/jovenes.pdf. [Acceso: 12 de julio de 2012].

2. Míguez HA. Alcohol y disponibilidad en la fiesta adolescente. Trastor adict 2009;11(1):39-43.

3. Míguez HA. El espejo de Erised. Determinaciones sociales del abuso epidémico de alcohol. Ed. Programa de Epidemiología Psiquiátrica. Buenos Aires, 2005. Disponible en http://miguezhugo.com.ar/ERI/eri.pdf. [Acceso: 6 de julio de 2012].

4. Varela A, Pritchard ME. Peer influence: use of alcohol, tobacco, and prescription medications. J Am Coll Health 2011;59(8):751-6.

5. Braschi ME, Santos IJ. Drogas y adolescencia. Rev Hosp Niños B Aires 2006;48(220):281-91.

6. Petroianu A, Reis DC, Cunha BD, Souza DM. Prevalence of alcohol, tobacco and psychotropic drug use among medical students at the Universidade Federal de Minas Gerais. Rev Assoc Med Bras 2010;56(5):568-71.

7. Romero MI, Santander J, Hitschfeld MJ, Labbé M, et al.
Consumo de sustancias ilícitas y psicotrópicos entre los estudiantes de medicina de la Pontificia Universidad Católica de Chile. Rev Med Chile 2009;137:459-65.

8. Caamaño-Isorna F, Mota N, Crego A, Corral M, et al. Consumption of medicines, alcohol, tobacco and cannabis among university students: a 2-year follow-up. Int J Public Health 2011;56(3):247-52.

9. Hammond D, Ahmed R, Yang WS, Brukhalter R, et al. Illicit substance use among Canadian youth: trends between 2002 and 2008. Can J Public Health 2011;102(1):7-12.

10. Cabrerizo S, Docampo PC. Alcohol y mezcla de fármacos: modalidad de abuso frecuente. Arch Argent Pediatr 2010;108(5):e111-3.

11. Yip L. Ethanol. En: Flomenbaum NE, Goldfrank LR, Hoffman RS, et al., eds. Golfrank's toxicologic emergencies. $8^{\text {th }}$ ed. New York: McGraw-Hill; 2006. Págs.1147-61.

12. Ellenhorn MJ. Alcohols and glycols. En: Ellenhorn MJ, Schonwald S, Ordog G, et al., eds. Ellenhorn's medical toxicology: Diagnosis and treatment of human poisoning. $2^{\text {nd }}$ ed. Baltimore: Williams and Wilkins; 1997. Págs.1127-45.

13. Argentina. SEDRONAR. Estudio Nacional sobre consumo en estudiantes de enseñanza media. Informe preliminar 2011: Dirección Nacional del Observatorio Argentino de Drogas. Disponible en: http:/ /www.marcelogastaldi.com. ar/archivos/novedades_MG/archivos/12-05-08-Estudio NacionalConsumoEstudiantesEnsMedia.pdf. [Acceso: 10 de agosto de 2012].

14. Instituto Nacional de Estadística y Censos. Anexo a los resultados de la Encuesta Nacional sobre Prevalencias de Consumo de Sustancias Psicoactivas 2008-ENPreCoSP-2008. Buenos Aires, 2008. Disponible en: http://www.indec.gov.ar/ftp/cuadros/publicaciones / anexo_enprecosp_29_07_08.pdf. [Acceso: 27 de junio de 2014].

15. Argentina. Ministerio de Salud de la Nación. Sistema de Vigilancia Epidemiológica en Salud Mental y Adicciones. Algunos datos sobre el consumo de alcohol en Argentina. Año 2011. Buenos Aires, 2011. Disponible en: http://www. msal.gov.ar/saludmental/images/stories/info-equipos/ pdf/4-algunos-datos-sobre-el-consumo-de-alcohol.pdf. [Acceso: 10 de agosto de 2012].

16. SchmidtV.Predictores de abuso de alcohol en adolescentes. Mitos versus evidencia empírica. Anu investig (Fac Psicol Univ B Aires) 2007;14:229-39.

17. Míguez HA. Patrones culturales de la alcoholización social en estudiantes bonaerenses. Vertex (B Aires) 2009;XX(87):325-8. 
ANEXO

\section{ENCUESTA}

\section{Marcá con una cruz la respuesta que considerás más adecuada.}

Edad:

Sexo: Femenino

Masculino

1. ¿Qué bebida consumís habitualmente cuando estás con tus amigos?

Vino

Bebidas energizantes (Speed, etc.)

Cerveza

Agua, jugos y gaseosas

Vodka, gin, whisky, ron, tequila, grapa

Otros: fernet, Gancia, licores

2. ¿Qué cantidad tomás cuando salís los fines de semana?

No tomo alcohol

1 lata de cerveza $(330 \mathrm{ml})$ o 1 vaso de vino $(200 \mathrm{ml})$ o 1 trago $(30-50 \mathrm{ml})$ de fernet, ron, ginebra, vodka, whisky

Más de una lata de cerveza, más de 1 vaso de vino (200 ml) o más de 1 trago (30-50 ml) de fernet, ron, ginebra, vodka, whisky

3. ¿Qué cantidad de alcohol tomás habitualmente (todos los días)?

No tomo alcohol

Hasta 1 lata de cerveza $(330 \mathrm{ml})$ o 1 vaso de vino $(200 \mathrm{ml})$ o 1 trago $(30-50 \mathrm{ml})$ de fernet, ron, ginebra, vodka, whisky

Más de una lata de cerveza, más de 1 vaso de vino (200 ml) o más de 1 trago (30-50 ml) de fernet, ron, ginebra, vodka, whisky

4. ¿Con qué frecuencia tomás bebidas alcohólicas habitualmente?

No tomo alcohol

Todos los días

De 2 a 3 veces por semana

1 vez por semana

1 vez cada 15 días

1 vez al mes

5. ¿A qué edad comenzaste a probar alcohol?

6. ¿Alguna vez mezclaste medicamentos o pastillas con alcohol?

Sí $\square \quad$ No

7. ¿A qué edad comenzaste a probar alcohol y medicamentos?

8. ¿Sabés si tus amigos probaron o consumieron medicamentos o pastillas mezcladas con alcohol alguna vez? Sí $\square$ No

9. ¿Sabés cuál es el uso común de esas pastillas?

No sé para qué se usan

Tranquilizantes (medicación para dormir)

Relajantes musculares

Hipoglucemiantes (para controlar el azúcar en la sangre o la diabetes)

Medicación psiquiátrica

Analgésicos (medicación para el dolor) 
10. En caso de que hayas probado pastillas con alcohol, ¿cómo las conseguiste?

De tu casa o la casa de tus amigos

Les convidaron

Las compraron

De otra manera

No sé

11. ¿En dónde tomás alcohol habitualmente?

No tomo alcohol

Tu casa o la casa de algún amigo

Boliche

Escuela

Espacio público (plaza, club, calle, etc.)

12. ¿Alguna vez experimentaste alguno de los siguientes sintomas luego de haber consumido alcohol?

Mareos, náuseas, vómitos, cefalea y/o dolor abdominal. Sí No

Pérdida de conciencia (no recordás lo que te pasó, te quedaste durmiendo en la vía pública, etc.) o convulsiones. Sí No

Nunca me pasó. Sí No

13. ¿Alguna vez tuviste que recibir ayuda médica o permanecer en observación en guardia en algún centro de salud luego de haber tomado alcohol?

Nunca

1 vez

De 2 a 3 veces

Más de 3 veces

14. ¿Alguna vez tuviste que recibir ayuda médica o permanecer en observación en guardia en algún centro de salud luego de haber tomado alcohol y pastillas?

Nunca

1 vez

De 2 a 3 veces

Más de 3 veces

15. ¿Podés salir o reunirte con tus amigos y divertirte sin tomar alcohol?

Sí $\square \quad$ No

16. En caso de que tomes alcohol, ¿qué crees que opinan tus padres al respecto?

Lo aprueban

Lo desaprueban

No opinan

Lo desconocen

17. En caso de que tomes alcohol, ¿lo saben tus padres?

Sí $\square \quad$ No

18. ¿Para qué consumís alcohol y/o medicamentos?

No consumo alcohol ni medicamentos

Para divertirme

Porque los demás lo hacen

Para compartir algo con mis amigos

Para evadirme y olvidar problemas

No sé por qué lo hago 